\title{
SOME EXPLICIT CASES OF THE SELBERG TRACE FORMULA FOR VECTOR VALUED FUNCTIONS
}

\author{
JEFFREY STOPPLE
}

\begin{abstract}
The trace formula for $S L(2, \mathbf{Z})$ can be developed for vector-valued functions which satisfy an automorphic condition involving a group representation $\pi$. This paper makes this version explicit for the class of representations which can be realized as representations of the finite group $\operatorname{PSL}(2, \mathbf{Z} / q)$ for some prime $q$. The body of the paper is devoted to computing, for the singular representations $\pi$, the determinant of the scattering matrix $\Phi(s, \pi)$ on which the applications depend. The first application is a version of the Roelcke-Selberg conjecture. This follows from known results once the scattering matrix is given.

The study of representations of $S L(2, \mathbf{Z})$ in finite-dimensional vector spaces of (scalar-valued) holomorphic forms dates back to Hecke. Similar problems can be studied for vector spaces of Maass wave forms, with fixed level $q$ and eigenvalue $\lambda$. One would like to decompose the natural representation of $S L(2, \mathbf{Z})$ in this space, and count the multiplicities of its irreducible components. The eigenvalue estimate obtained for vector-valued forms is equivalent to an asymptotic count, as $\lambda \rightarrow \infty$, of these multiplicities.
\end{abstract}

The trace formula for $S L(2, \mathbf{Z})$ can be developed for vector-valued functions which satisfy an automorphic condition involving a group representation $\pi$. In fact, Selberg's original paper stated the trace formula in this generality. This paper makes this version explicit for the class of representations which can be realized as representations of the finite group $\operatorname{PSL}(2, \mathbf{Z} / q)$ for some prime $q$. We then present some applications. The body of the paper is devoted to computing, for the singular representations $\pi$, the determinant of the scattering matrix $\Phi(s, \pi)$ on which the applications depend. The Dirichlet series coefficients of $\Phi(s, \pi)$ are matrix coefficient functions for $\pi$. To compute the scattering matrix one must first classify the singular representations and determine the matrix coefficients. By using the Frobenius reciprocity theorem, we show that the singular representations are essentially the principal series for the finite group, i.e., induced from characters on the subgroup of upper triangular matrices. Matrix coefficients for the principal series are easily computed. The scattering matrix is then summed by standard number theoretic techniques. The first application is a version of the Roelcke-Selberg conjecture; i.e., an estimate on the asymptotics of the eigenvalues for the Laplace operator in the relevant

Received by the editors December 26, 1987 and, in revised form, April 25, 1988.

1980 Mathematics Subject Classification (1985 Revision). Primary 11 F72.

Key words and phrases. Scattering matrix, Roelcke-Selberg conjecture, Maass wave form. 
Hilbert space. This follows from known results once the scattering matrix is given.

The study of representations of $S L(2, \mathbf{Z})$ is finite-dimensional vector spaces of (scalar-valued) holomorphic forms dates back to Hecke, who found a connection to class numbers of imaginary quadratic fields. Similar problems can be studied for vector spaces of Maass save forms, with fixed level $q$ and eigenvalue $\lambda$. One would like to decompose the natural representation of $S L(2, \mathbf{Z})$ in this space, and count the multiplicities of its irreducible components. The eigenvalue estimate obtained for vector-valued forms is equivalent to an asymptotic count, as $\lambda \rightarrow \infty$, of these multiplicities.

Most of this work is contained in the author's Ph.D. dissertation. The author would like to thank Professors Audrey Terras, Harold Stark and James Hafner for their support and encouragement.

\section{THE TRACE FORMULA}

We need some background on the trace formula, as in [9] and [2].

Suppose $\pi$ is a unitary finite-dimensional representation of $\Gamma=S L(2, \mathbf{Z})$ by matrices, i.e., a homomorphism $\pi: \Gamma \rightarrow U(n)$. Let $L^{2}(\Gamma, \pi)$ be the Hilbert space of functions $F: H \rightarrow \mathbf{C}^{n}$ satisfying

(i) $F(\gamma z)=\pi(\gamma) F(z), \gamma \in \Gamma, z \in H$,

(ii) $\langle F, F\rangle<\infty$ where $\langle F, G\rangle=\int_{\mathscr{F}} F(z)^{t} \overline{G(z)} d x d y / y^{2}$.

Here $H$ is the upper half plane and $\mathscr{F}$ is a fundamental domain for $\Gamma$. The volume $\mu$ of the domain is $\pi / 3$. Since $\pi$ is unitary there are orthonormal vectors $f_{1}, \ldots, f_{n}$ in $\mathbf{C}^{n}$ that diagonalize the matrix $\pi\left[\begin{array}{ll}1 & 1 \\ 0 & 1\end{array}\right]$; so $\pi\left[\begin{array}{ll}1 & 1 \\ 0 & 1\end{array}\right] f_{j}=$ $\exp \left(2 \pi i \alpha_{j}\right) f_{j}$ for some $\alpha_{j}, 0 \leq \alpha_{j}<1$. We can assume that $f_{j}$ are ordered so that the $\alpha_{j}$ are increasing. Let $c_{\pi}$ be the number of times $\alpha_{j}=0$ occurs, i.e., the multiplicity of 1 as an eigenvalue for $\pi\left[\begin{array}{ll}1 & 1 \\ 0 & 1\end{array}\right]$. The representation is singular if $c_{\pi}>0$, and nonsingular otherwise. $c_{\pi}$ is called the degree of singularity of $\pi$. It counts the multiplicity of the continuous spectrum. The vector space spanned by $\left\{f_{1}, \ldots, f_{c_{\pi}}\right\}$ is denoted $J(\pi)$, for Jacquet module.

A generic representation will usually be called $\pi$. Unfortunately, the same symbol will denote the numerical constant $\pi$.

We can now define a vector-valued Eisenstein series. If $1 \leq h \leq c_{\pi}$, let

$$
E\left(z, s, \pi, f_{h}\right)=\sum_{\gamma \in \Gamma_{\infty} \backslash \Gamma}\left(\pi\left(\gamma^{-1}\right) f_{h}\right) \operatorname{Im}(\gamma z)^{s},
$$

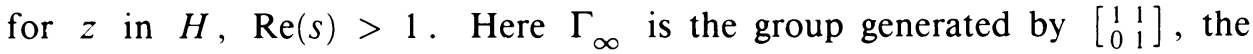
stabilizer of $\infty$. Let $M$ be the unitary matrix whose columns are the vectors $f_{1}, \ldots, f_{n}$. Then the complex conjugate of the entry in row $i$, column $j$ of $M^{*} \pi(\gamma) M$ is just $\left\langle f_{i}, \pi(\gamma) f_{j}\right\rangle=f_{i}^{t} \bar{\pi}(\gamma) \overline{f_{j}}$. We can define a Dirichlet series 
based on these matrix coefficient functions. For $i \leq h, p \leq c_{\pi}, \operatorname{Re}(s)>1$, let

$$
\begin{aligned}
\phi_{h, p}(s)=\sqrt{\pi} \frac{\Gamma\left(s-\frac{1}{2}\right)}{\Gamma(s)} & \sum_{\substack{\gamma \in \Gamma \infty \backslash \Gamma / \Gamma_{\infty} \\
\gamma=\left[\begin{array}{ll}
a & b \\
c & d
\end{array}\right], c \neq 0}}\left\langle f_{h}, \pi(\gamma) f_{p}\right\rangle \frac{1}{|c|^{2 s}} .
\end{aligned}
$$

These functions are actually the coefficients of the $y^{1-s}$ terms in the Fourier expansions of the Eisenstein series. The $c_{\pi}$ by $c_{\pi}$ matrix $\left\{\phi_{h, p}(s)\right\}$ is called the scattering matrix $\Phi(s, \pi)$, and its determinant is called the scattering determinant.

\section{CONGRUENCE REPRESENTATIONS}

For applications, we would like to compute the scattering matrix when it exists $\left(c_{\pi}>0\right.$ in the previous notation). The most general representations $\pi$ for which we can do this have the property that the kernel of $\pi$ as a group homomorphism contains a congruence subgroup $\Gamma(q)$. Then $\pi$ defines a representation of the finite factor group $\Gamma / \Gamma(q) \cong \operatorname{PSL}(2, \mathbf{Z} / q)$. Representations of $\Gamma$ of this type will be called congruence representations. For convenience we will also assume the level $q$ is prime. The more general case of square free level is treated in [11].

We can think of the scattering matrix $\Phi(s, \pi)$ as a matrix of Dirichlet series, or as a Dirichlet series with matrix coefficients

$$
\Phi(s, \pi)=\sqrt{\pi} \frac{\Gamma\left(s-\frac{1}{2}\right)}{\Gamma(s)} \sum_{\substack{\gamma \in \Gamma \infty \mid \Gamma / \Gamma_{\infty} \\
\gamma=\left[\begin{array}{ll}
a & b \\
c & d
\end{array}\right], c \neq 0}} A_{\gamma}^{\pi} \frac{1}{|c|^{2 s}},
$$

where $A_{\gamma}^{\pi}$ is the matrix with entries $\left\langle f_{h}, \pi(\gamma) f_{p}\right\rangle$ for $1 \leq h, p \leq c_{\pi}$ and the matrix $M=\left[f_{1}\left|f_{2}\right| \cdots \mid f_{n}\right]$ is a unitary matrix that diagonalizes $\pi\left[\begin{array}{ll}1 & 1 \\ 0 & 1\end{array}\right]$. Thus

$$
M^{*} \pi\left[\begin{array}{ll}
1 & 1 \\
0 & 1
\end{array}\right] M=\left[\begin{array}{cc}
I_{c_{\pi}} & 0 \\
0 & D
\end{array}\right]
$$

and $D$ is an $n-c_{\pi}$ by $n-c_{\pi}$ diagonal matrix, with no eigenvalues of 1 . The complex conjugate of $A_{\gamma}^{\pi}$ is just the singular matrix of $M^{*} \pi(\gamma) M$. By this we mean

$$
M^{*} \pi(\gamma) M=\left[\begin{array}{cc}
\bar{A}_{\gamma}^{\pi} & * \\
* & *
\end{array}\right] .
$$

Two representations are said to be equivalent if they differ by a conjugation. Thus $M^{*} \pi M$ is equivalent to $\pi$ as $M$ is unitary. Since the matrix $M$ is not unique, $\Phi(s, \pi)$ is not well defined.

Lemma 1. $\Phi(s, \pi)$ is well defined up to conjugation by a matrix of constants, and depends only on the equivalence class of $\pi$.

Proof. By its definition in terms of the coefficients of the equivalent representation $M^{*} \pi M$, it is clear that $\Phi(s, \pi)$ will depend only on the equivalence class 
of $\pi$. Suppose $\pi_{1}$ and $\pi_{2}$ are both equivalent to $\pi$, with

$$
\pi_{1}\left[\begin{array}{ll}
1 & 1 \\
0 & 1
\end{array}\right]=\left[\begin{array}{cc}
I & 0 \\
0 & D_{1}
\end{array}\right] \text { and } \pi_{2}\left[\begin{array}{ll}
1 & 1 \\
0 & 1
\end{array}\right]=\left[\begin{array}{cc}
I & 0 \\
0 & D_{2}
\end{array}\right] .
$$

Then $\pi_{1}$ and $\pi_{2}$ are equivalent to each other so there exists a matrix

$$
N=\left[\begin{array}{ll}
N_{11} & N_{12} \\
N_{21} & N_{22}
\end{array}\right]
$$

with $\pi_{1}(\gamma) N=N \pi_{2}(\gamma)$ for all $\gamma$. Take $\gamma=\left[\begin{array}{ll}1 & 1 \\ 0 & 1\end{array}\right]$ to see that $N_{12}=N_{12} D_{2}$ and $D_{1} N_{21}=N_{21} . I-D_{2}$ and $D_{1}-I$ are invertible since neither $D_{1}$ nor $D_{2}$ has eigenvalue 1 by definition, so $N_{12}=0$ and $N_{21}=0$. Thus

$$
\text { if } \pi_{1}(\gamma)=\left[\begin{array}{cc}
\bar{A}_{\gamma} & * \\
* & *
\end{array}\right] \quad \text { then } \pi_{2}(\gamma)=N^{-1} \pi_{1}(\gamma) N=\left[\begin{array}{cc}
N_{11}^{-1} \bar{A}_{\gamma} N_{11} & * \\
* & *
\end{array}\right]
$$

and so it follows that

$$
\Phi\left(s, \pi_{2}\right)=\Phi\left(s, N^{-1} \pi_{1} N\right)=N_{11}^{-1} \Phi\left(s, \pi_{1}\right) N_{11} .
$$

To study the scattering matrix we need to first determine the singular representations and compute the matrix coefficients. The rest of this section contains the solution of this algebraic problem. Many details are omitted, as they are easily filled in and are similar to computations found in [6]. The full calculations are found in [11]. Lemma 1 is crucial because it means we can compute matrix coefficients in any convenient realization of $\pi$ so long as we choose, as basis for the space $\pi$ acts in, eigenvectors of $\pi\left[\begin{array}{ll}1 & 1 \\ 0 & 1\end{array}\right]$. We will use this fact repeatedly, without explicit reference to the lemma.

2.1. Singular representations. Fix a prime $q$ and let $G=S L(2, \mathbf{Z} / q)$. Two subgroups of $G$ will play an important role.

$$
\begin{gathered}
B=\left\{g \in G \mid g=\left[\begin{array}{cc}
\alpha^{-1} & \beta \\
0 & \alpha
\end{array}\right] \text { for } \alpha \in \mathbf{Z} / q^{*}, \beta \in \mathbf{Z} / q\right\}, \\
U=\left\{g \in G \mid g=\left[\begin{array}{cc}
1 & \beta \\
0 & 1
\end{array}\right] \text { for } \beta \in \mathbf{Z} / q\right\} .
\end{gathered}
$$

A representation $\pi$ of $G$ is singular if and only if the trivial representation is contained in the restriction of $\pi$ to $U$. This important fact allows us to use the machinery of induction and restriction of representations. For example, every Dirichlet character $\chi \bmod q$ defines a representation of $B$ by

$$
\chi\left[\begin{array}{cc}
\alpha^{-1} & \beta \\
0 & \alpha
\end{array}\right]=\chi(\alpha)
$$

We will use the same symbol $\chi$ in both senses. Each such representation determines an induced representation of $G$, denoted $\hat{\chi}$. These representations are sometimes called the principal series of $G$ in analogy to the Lie groups case. The action of $\hat{\chi}$ is by right translation in the function space

$$
\{f: G \rightarrow C \mid f(b g)=\chi(b) f(g) \text { for } g \in C, b \in B\} .
$$


Lemma 2. Let $\chi$ be any Dirichlet character $\bmod q$, and $\hat{\chi}$ the induced representation. Then $\hat{\chi}$ is singular and $c_{\hat{\chi}}$ is equal to 2 .

Proof. We want to compute the restriction to $U$ of a representation induced from $B$. The Induction Restriction Theorem (see [10]) gives the result.

Lemma 3. Let $\pi$ be any irreducible representation of $G$. Then $\pi$ is singular $\left(c_{\pi}>0\right)$ if and only if there is a character $\chi$ such that $\pi$ is a subrepresentation of $\hat{\chi}$.

Proof. This is an application of the Frobenius Reciprocity Theorem (see [10]).

Recall we defined $J(\pi)$ as the space of vectors fixed by $\pi\left[\begin{array}{ll}1 & 1 \\ 0 & 1\end{array}\right]$. In the case when $\pi$ is a subrepresentation of $\hat{\chi}$ it is easy to see that $J(\pi)$ is contained in $J(\hat{\chi})$ and so $c_{\pi} \leq 2$ by Lemma 1 .

Lemma 4. Again let $\chi$ be any character. Then $\hat{\chi}$ is irreducible if and only if $\chi$ is not a real-valued character. If $\chi$ is real then $\hat{\chi}$ decomposes as a direct sum of two inequivalent irreducible representations.

Proof. We apply Mackey's irreducibility criterion, which is just a combination of Induction-restriction and Frobenius reciprocity.

The lemmas can be summarized as follows. There are two real characters $\chi$ of $\mathbf{Z} / q^{*}$ for $q$ odd. One of them is the trivial character 1 . By Frobenius reciprocity the trivial representation of $G$ is contained in $\hat{1}$. All principal series representations of $G$ have dimension $[G: B]=q+1$, so that other irreducible component of $\hat{1}$, call it $\theta$, is $q$-dimensional. For the quadratic character $\chi_{1}$ we will later see that both of the two irreducible components of $\hat{\chi}_{1}$ are $(q+1) / 2-$ dimensional. It is easily verified that for any representations $\pi$ and $\theta$ of $G$, $J(\pi \oplus \theta)=J(\pi) \oplus J(\theta)$ and so $c_{\pi \oplus \theta}=c_{\pi}+c_{\theta}$. Each of the four representations mentioned above is singular by Lemma 3 . On the other hand, none can have degree of singularity greater than 1 , since the degree of singularity of the sum of the two components (i.e., the induced representation) is 2 by Lemma 2. Thus each of these four representations has degree of singularity equal 1 . There are $(q-3)$ characters $\chi$ of $\mathbf{Z} / q^{*}$ which are not real valued. It can be shown that for these $\hat{\chi}$ is equivalent to $\left(\hat{\chi}^{-1}\right)$, but this fact will not be needed. Each of these representations is irreducible, by Lemma 4 , and has degree of singularity equal 2 by Lemma 2. By Lemma 3, the remaining irreducible representations of $G$ (there are $(q+3) / 2$ of them) are all nonsingular. The case of the prime 2 can be handled similarly. Of course, the only Dirichlet character mod 2 is the trivial character.

A representation $\pi$ of $S L(2, \mathbf{Z} / q)$ is said to be even if it projects to a representation of $\operatorname{PSL}(2, \mathbf{Z} / q)$ i.e., $\pi\left[\begin{array}{cc}-1 & 0 \\ 0 & -1\end{array}\right]$ is the identity. The singular even representations can be classified by considering representations induced from characters $\chi$ on the subgroup $B$. These are realized in a vector space of functions on the group with the action by right translation. Thus the induced representation $\hat{\chi}$ is even if and only if, for all functions $f$ in the space and all 
$g$ in the group,

$$
f(g)=f\left(g\left[\begin{array}{cc}
-1 & 0 \\
0 & -1
\end{array}\right]\right)=f\left(\left[\begin{array}{cc}
-1 & 0 \\
0 & -1
\end{array}\right] g\right)=\chi(-1) f(g) .
$$

Thus $\hat{\chi}$ is even if the character $\chi$ is even, and $\hat{\chi}\left[\begin{array}{cc}-1 & 0 \\ 0 & -1\end{array}\right]$ is -1 times the identity map if the character $\chi$ is odd.

2.2 Matrix coefficients. We must now compute matrix coefficients for each singular representation $\pi$ of prime level $q$. The coefficients correspond to basis elements for $J(\pi)$. This gives, in the notation of section one, the matrix $A_{g}^{\pi}$ for each element $g$ in the group. Matrix coefficients for this group have been calculated before, in [4] for example. We need coefficients with respect to a specific basis, so it is easiest to start from scratch.

Consider first the case of $\hat{\chi}$, where $\chi$ is not real. The action of $\hat{\chi}$ is by right translation in the function space

$$
\{f: G \rightarrow C \mid f(b g)=\chi(b) f(g) \text { for } g \in G, b \in B\} .
$$

Since $\chi(u)=1$ for $u$ in $U$, these functions can be thought of as functions on the cosets $U \backslash G$. A different realization of $\hat{\chi}$ will be helpful. Let $F$ be a two-dimensional vector space over $\mathbf{Z} / q$ with the origin deleted. Then $G$ acts transitively on $F$ by

$$
(x, y)\left[\begin{array}{ll}
\alpha & \beta \\
\gamma & \delta
\end{array}\right]=(\alpha x+\gamma y, \beta x+\delta y) .
$$

The subgroup $U$ is the stabilizer of the point $(0,1)$, so $F$ is equivalent to $U \backslash G$ as $G$ spaces. The induced representation $\hat{\chi}$ is equivalent to the action of $G$ in the vector space

$$
\left\{f: F \rightarrow \mathbf{C} \mid f(\lambda x, \lambda y)=\chi(\lambda) f(x, y) \text { for } \lambda \in(\mathbf{Z} / q)^{*}\right\}
$$

by $\hat{\chi}\left[\begin{array}{ll}\alpha & \beta \\ \gamma & \delta\end{array}\right] f(x, y)=f(\alpha x+\gamma y, \beta x+\delta y)$. A function $f$ is determined by its values on the points $(1, x)$ for $x \in \mathbf{Z} / q$ and $(0,1)$. For convenience call the values of the function $f(x)$ and $f(\infty)$ respectively. In this notation the action of $\hat{\chi}$ is as follows:

$$
\begin{aligned}
& \left.\begin{array}{l}
\hat{\chi}(g) f(x)=\chi(\gamma x+\alpha) f\left(\frac{\delta x+\beta}{\not x+\alpha}\right) \quad \text { if } x \neq \frac{-\alpha}{\gamma^{\prime}} \\
\hat{\chi}(g) f\left(\frac{-\alpha}{i^{\prime}}\right)=\chi\left(\frac{-1}{i^{\prime}}\right) f(\infty) \\
\hat{\chi}(g) f(\infty)=\chi(\gamma) f\left(\frac{\delta}{i^{\prime}}\right)
\end{array}\right\} \\
& \text { for } g=\left[\begin{array}{ll}
\alpha & \beta \\
\gamma & \delta
\end{array}\right], \gamma \not \equiv 0 \bmod q \text {, } \\
& \left.\begin{array}{l}
\hat{\chi}(g) f(x)=\chi(\alpha) f\left(\frac{\alpha^{-1} x+\beta}{\alpha}\right) \\
\hat{\chi}(g) f(\infty)=\chi\left(\alpha^{-1}\right) f(\infty)
\end{array}\right\} \quad \text { for } g=\left[\begin{array}{cc}
\alpha & \beta \\
0 & \alpha^{-1}
\end{array}\right] \text {. }
\end{aligned}
$$

Introduce an inner product on the vector space by defining

$$
\left\langle f_{1}, f_{2}\right\rangle=\sum_{x \in \mathbf{Z} / q} f_{1}(x) \overline{f_{2}(x)}+f_{1}(\infty) \overline{f_{2}(\infty)}
$$


Considering these functions as vectors in $C^{q+1}$, this is the usual inner product. Let

$$
f_{1}(x)=\frac{1}{\sqrt{q}}, \quad f_{1}(\infty)=0,
$$

and

$$
f_{2}(x)=0, \quad f_{2}(\infty)=1 .
$$

Then $\hat{\chi}\left[\begin{array}{ll}1 & 1 \\ 0 & 1\end{array}\right] f_{1}=f_{1}$ and $\hat{\chi}\left[\begin{array}{ll}1 & 1 \\ 0 & 1\end{array}\right] f_{2}=f_{2}$ by (2.2) above. Thus $\left\{f_{1}, f_{2}\right\}$ is an orthonormal basis for $J(\hat{\chi})$. We must compute four matrix coefficients in each of two cases.

(i) If $g=\left[\begin{array}{ll}\alpha & \beta \\ \gamma & \delta\end{array}\right]$ and $\gamma \not \equiv 0 \bmod q$, then

$$
\begin{aligned}
& \hat{\chi}(g) f_{1}(x)=\frac{\chi(\gamma x+\alpha)}{\sqrt{q}} \text { for } x \neq \frac{-\alpha}{\gamma}, \\
& \hat{\chi}(g) f_{1}\left(\frac{-\alpha}{\gamma}\right)=0, \\
& \hat{\chi}(g) f_{1}(\infty)=\frac{\chi(\gamma)}{\sqrt{q}}
\end{aligned}
$$

and

$$
\begin{aligned}
& \hat{\chi}(g) f_{2}(x)=0 \quad \text { for } x \neq \frac{-\alpha}{\gamma}, \\
& \hat{\chi}(g) f_{2}\left(\frac{-\alpha}{\gamma}\right)=\chi\left(\frac{-1}{\gamma}\right)=\chi(-1) \bar{\chi}(\gamma), \\
& \hat{\chi}(g) f_{2}(\infty)=0
\end{aligned}
$$

by applying (2.1) above. Thus

$$
\begin{aligned}
& \left\langle f_{1}, \hat{\chi}(g) f_{1}\right\rangle=\sum_{x \neq-\alpha / \gamma} \frac{\hat{\chi}(\gamma x+\alpha)}{q}=0, \\
& \left\langle f_{2}, \hat{\chi}(g) f_{1}\right\rangle=\frac{\bar{\chi}(\gamma)}{\sqrt{q}}, \\
& \left\langle f_{1}, \hat{\chi}(g) f_{2}\right\rangle=\frac{\chi(-1) \chi(\gamma)}{\sqrt{q}}, \\
& \left\langle f_{2}, \hat{\chi}(g) f_{2}\right\rangle=0 .
\end{aligned}
$$

These computations give the matrix $A_{g}^{\hat{\chi}}$ in the notation of $\S 1$ :

(ii) If $g=\left[\begin{array}{cc}\alpha & \beta \\ 0 & \alpha^{-1}\end{array}\right]$ then

$$
\left[\begin{array}{cc}
0 & \frac{\chi(y)}{\sqrt{q}} \\
\frac{\bar{\chi}(y)}{\sqrt{q}} & 0
\end{array}\right]
$$

$$
\begin{aligned}
\hat{\chi}(g) f_{1}(x) & =\frac{\chi(\alpha)}{\sqrt{q}}, \\
\hat{\chi}(g) f_{1}(\infty) & =0, \\
\hat{\chi}(g) f_{2}(x) & =0, \\
\hat{\chi}(g) f_{2}(\infty) & =\chi\left(\alpha^{-1}\right)=\bar{\chi}(\alpha)
\end{aligned}
$$


by applying (2.2) above. Thus

$$
\begin{aligned}
& \left\langle f_{1}, \hat{\chi}(g) f_{1}\right\rangle=\sum_{x \in \mathbf{Z} / q} \frac{\bar{\chi}(\alpha)}{q}=\bar{\chi}(\alpha), \\
& \left\langle f_{2}, \hat{\chi}(g) f_{1}\right\rangle=0, \\
& \left\langle f_{1}, \hat{\chi}(g) f_{2}\right\rangle=0, \\
& \left\langle f_{2}, \hat{\chi}(g) f_{2}\right\rangle=\chi(\alpha) .
\end{aligned}
$$

So for this $g$ we get that $A_{g}^{\hat{\chi}}$ is

$$
\left[\begin{array}{cc}
\bar{\chi}(\alpha) & 0 \\
0 & \chi(\alpha)
\end{array}\right]
$$

Next consider the trivial character. The induced representation $\hat{1}$ decomposes into two inequivalent components. One of them must be the trivial representation by Frobenius reciprocity. The matrix coefficients for the trivial representation are easy to compute: they are always equal to one. By Lemma 4 above, the other, $q$-dimensional component of $\hat{1}$ is irreducible. Denote by $\theta$ the representation obtained. The action of $\theta$ is computed as in the monomial case, by (2.1) and (2.2) above. The details are omitted. The matrix coefficients are listed in Table 1 below.

In the case of the quadratic character $\chi_{1}$ the induced representation is again reducible, but the decomposition into irreducibles is somewhat complicated. It is necessary to introduce the Fourier transform on $\mathbf{Z} / q$ and consider functions $f$ such that $\hat{f}$ vanishes on the squares in $\mathbf{Z} / q$. The restriction of $\hat{\chi}_{1}$ to this space is denoted $\pi^{+}$, and the other component is denoted $\pi^{-}$. The details are outlined in [13] and the full computation is in [11]. The matrix coefficients for $\pi^{+}$and $\pi^{-}$are in Table 1 .

\section{Summing the SCATTERING MATRIX}

We will compute the scattering matrix $\Phi(s, \pi)$ in the case when $\pi=\hat{\chi}$ is monomial. The other cases are similar. We first compute the sum of the matrix valued Dirichlet series:

$$
\sum_{g \in \Gamma_{\infty} \backslash \Gamma / \Gamma_{\infty}} A_{g}^{\pi} \frac{1}{|c|^{2 s}} .
$$

We will choose the usual double coset representatives.

Lemma. For $c>0,0 \leq d<c$, with $(c, d)=1$, fix a matrix $g[c, d]$ in $S L(2, \mathbf{Z})$ with $g[c, d]=\left[\begin{array}{ll}a & b \\ c & d\end{array}\right]$ for some integers $a$ and $b$. Then

$$
\Gamma_{\infty} \backslash \Gamma / \Gamma_{\infty}-\Gamma_{\infty}=\bigcup_{c>0} \bigcup_{\substack{0 \leq d<c \\(d, c)=1}} \Gamma_{\infty} g[c, d] \Gamma_{\infty} .
$$

Proof. Straightforward calculation. 
For notational convenience, define $A[c, d]=A_{g[c, d]}^{\pi}$. We see that $(3.1)$ is equal to

$$
\sum_{c>0} \sum_{\substack{0 \leq d<c \\(d, c)=1}} A[c, d] \frac{1}{c^{2 s}}
$$

Lemma. If $(c, q)>1$, then

$$
\sum_{\substack{0 \leq d<c \\
(d, c)=1}} A[c, d]=\sum_{\substack{0 \leq d<c \\
(d, c)=1}}\left[\begin{array}{cc}
\chi(d) & 0 \\
0 & \bar{\chi}(d)
\end{array}\right]=0 .
$$

Proof. Here we are using the fact that $d \equiv a^{-1} \bmod q$ in replacing $a$ by $d$ and $\chi$ by $\bar{\chi}$. Note that $\chi$ is not trivial $\bmod c$ as it is not trivial $\bmod q$.

The lemma says that (3.2) above reduces to

$$
\sum_{\substack{c>0 \\(c, q)=1}} \phi(c) \frac{1}{c^{2 s}} A[c, d],
$$

where $\phi(c)$ is Euler's function. Since $(c, q)=1$, each of the matrices is antidiagonal.

We can now consider (3.3) above as an anti-diagonal matrix whose entries are Dirichlet series, i.e., (3.3) is equal to

$$
\frac{1}{\sqrt{q}}\left[\begin{array}{cc}
0 & \rho_{1}(s) \\
\rho_{2}(s) & 0
\end{array}\right]
$$

where, for $h=1,2$

$$
\rho_{h}(s)=\sum_{c>0} \phi(c) \frac{\psi_{h}(c)}{c^{2 s}}, \quad \psi_{1}=\chi, \psi_{2}=\bar{\chi}
$$

The sum is taken over all $c>0$ since $\psi_{h}$ is $\bmod q$ and the extra terms contribute nothing. We next compute a typical entry $\rho_{h}(s)$. For notational convenience we will suppress the subscript $h$.

Lemma. For $\psi$ as above,

$$
\sum_{n>0} \phi(n) \frac{\psi(n)}{n^{2 s}}=\prod_{\text {prime }} \frac{1-\psi(p) p^{-2 s}}{1-\psi(p) p^{1-2 s}}=\frac{L(2 s-1, \psi)}{L(2 s, \psi)} .
$$

Proof. This is a standard calculation with Dirichlet series.

The scattering matrix $\phi(s, \pi)$ is $\sqrt{\pi} \Gamma\left(s-\frac{1}{2}\right) / \Gamma(s)$ times the series considered above. Thus we have an anti-diagonal matrix

$$
\Phi(s, \pi)=\frac{\sqrt{\pi}}{\sqrt{q}} \frac{\Gamma\left(s-\frac{1}{2}\right)}{\Gamma(s)}\left[\begin{array}{cc}
0 & \rho_{1}(s) \\
\rho_{2}(s) & 0
\end{array}\right]
$$


by combining (3.4), (3.5) with the subscript re-introduced. Use the functional equation for $L(s, \chi)$ to replace the term $\Gamma\left(s-\frac{1}{2}\right) L(2 s-1, \chi)$ in each entry of (3.6) by

$$
(q / \pi)^{1 / 2}(q / \pi)^{1-2 s} \Gamma(1-s) L(2-2 s, \bar{\chi}) .
$$

We summarize what we proved in this section.

Theorem 1. Let $\chi$ a Dirichlet character $\bmod q, \chi \neq \bar{\chi}$, and $\hat{\chi}$ the representation of $\operatorname{PSL}(2, \mathbf{Z} / q)$ constructed above. Then

$$
\Phi(s, \hat{\chi})=\left(\frac{q}{\pi}\right)^{1-2 s} \frac{\Gamma(1-s)}{\Gamma(s)}\left[\begin{array}{cc}
0 & \frac{L(2-2 s), \bar{\chi})}{L(2 s) \chi)} \\
\frac{L(2-2 s, \bar{\chi})}{L(2 s, \bar{\chi})} & 0
\end{array}\right] .
$$

If $1, \theta, \pi^{+}$, and $\pi^{-}$are the other singular representations of $\operatorname{PSL}(2, \mathbf{Z} / q)$ constructed above, similar methods show

$$
\begin{aligned}
\Phi(s, 1) & =\pi^{2 s-1} \frac{\Gamma(1-s)}{\Gamma(s)} \frac{\zeta(2-2 s)}{\zeta(2 s)} \quad \text { (also computed in [2]), } \\
\Phi(s, \theta) & =\left(\frac{q}{\pi}\right)^{1-2 s} \frac{\Gamma(1-s)}{\Gamma(s)} \frac{\zeta(2-2 s)}{\zeta(2 s)} \frac{1-q^{2 s-2}}{1-q^{-2 s}} \\
\Phi\left(s, \pi^{+}\right) & =-\Phi\left(2, \pi^{-}\right)=\left(\frac{q}{\pi}\right)^{1-2 s} \frac{\Gamma(1-s)}{\Gamma(s)} \frac{\left.L(2-2 s), \chi_{1}\right)}{L\left(2 s, \chi_{1}\right)}
\end{aligned}
$$

\section{CONNections With MASS WAVE FORMS}

Let $\Delta$ be the non-Euclidean Laplace operator $y^{2}\left(\frac{\delta^{2}}{\delta x^{2}}+\frac{\delta^{2}}{\delta y^{2}}\right)$.

For a fixed representation $\rho$ of $\Gamma$ let

$$
\left.N(T, \rho)=\# \text { eigenvalues } \lambda=\left(\frac{1}{4}+r^{2}\right) \text { of } \Delta \text { in } L^{2}(\Gamma, \rho) \mid 0<r<T\right\} .
$$

It is shown in [2, p. 315] for more general groups than just $\Gamma=S L(2, \mathbf{Z})$ that

$$
N(t, \rho)-\frac{1}{4 \pi} \int_{-T}^{T} \frac{\phi^{\prime}}{\phi}\left(\frac{1}{2}+i r\right) d r \sim \operatorname{dim} \rho \frac{\mu}{4 \pi} T^{2}
$$

as $T \rightarrow \infty$. The function $\phi^{\prime} / \phi$ is the logarithmic derivative of the determinant of $\Phi(s, \rho)$. The constant $\mu$ denotes the measure of the fundamental domain, as in $\S 1$.

Corollary 1. Let $\pi$ be a congruence representation of prime level $q$. Then as $T \rightarrow \infty$ for $N(T, \pi)$ as above

$$
N(T, \pi) \sim(\operatorname{dim} \pi) \frac{\mu}{4 \pi} T^{2} .
$$

Proof. If there is no continuous spectrum (i.e., $c_{\pi}=0$ ) then the result follows from (4.1). In general we need to compute the determinant of the scattering matrix $\Phi(s, \pi)$ and show the contribution of the integral of the logarithmic derivative is negligible. By Theorem 7.1, p. 131 of [7] we now $\frac{L^{\prime}}{L}(1+2 i r, \chi)$ is $O(\log q(|2 r|+2))$ and $\frac{\Gamma^{\prime}}{\Gamma}\left(\frac{1}{2}+i r\right)$ is $O(\log |r|)$ by Stirling's formula. The 
terms $(q / \pi)^{-s}$ and $1-q^{-s}$ have logarithmic derivatives which, evaluated at $s=(1 / 2)+i r$, are $O(1)$. Integrating from $-T$ to $T$, the continuous spectrum contributes $O(T \log T)$ to the Weyl estimate, and the corollary is proved.

Corollary 1 has another interpretation. Suppose $f$ is a Maass wave form of level $q$, i.e., $f \in L^{2}(\Gamma(q), 1)$ and $\Delta f+\lambda f=0$ for some $\lambda$ in the notation of $\S 1$. If $\tau$ is any element of $\Gamma$ then $f(\tau z)$ satisfies the same properties that $f$ does. Thus $f(\tau z)$ is also a wave form of level $q$, for the same eigenvalue. For a fixed level and eigenvalue, we have a representation of $\Gamma$ in this finite dimensional vector space, by $f(z) \rightarrow f(\tau z)$. Clearly $\Gamma(q)$ is contained in the kernel of this representation. This gives a representation of the factor group $\Gamma / \Gamma(q)$, which is isomorphic to $\operatorname{PSL}(2, \mathrm{Z} / q)$. Such representations are completely reducible. The space of wave forms for level $q$ and fixed eigenvalue can thus be decomposed into a direct sum of spaces, with the action of $\operatorname{PSL}(2, \mathbf{Z} / q)$ irreducible in each. For a fixed irreducible representation $\pi$ of $\operatorname{PSL}(2, \mathbf{Z} / q)$ we would like to know the multiplicity of $\pi$ in this decomposition; i.e., for how many of the spaces is the action equivalent to $\pi$ ?

The corresponding question for holomorphic forms of weight $k$ was studied by Hecke, and answered explicitly for the case $k=2$ in [1, p. 731]. He also showed that for a prime level $p \equiv 3 \bmod 4$, the difference between the multiplicities for two specific representations was equal to the class number of the imaginary quadratic field $\mathbf{Q}(\sqrt{-p})$.

The study of this problem for Maass wave forms was motivated by a paper of Randol [8] in which he noted that in the case of a prime level $q$, nontrivial representations must occur for infinitely many eigenvalues $\lambda$. Thus there are infinitely many eigenvalues for $\Delta$ in $L^{2}(\Gamma(q), 1)$ which are not simple. It is conjectured that all eigenvalues for $\Delta$ in $L^{2}(\Gamma, 1)$ are simple.

Fix an irreducible $\pi$ of $\operatorname{PSL}(2, \mathbf{Z} / q)$. Let $m(\pi, \lambda)$ be the multiplicity of $\pi$ in the space of wave forms for level $q$ and eigenvalue $\lambda$. Huber has proved a lemma in [3] which says that $m(\pi, \lambda)$ is equal to the multiplicity of $\lambda$ as an eigenvalue in $L^{2}(\Gamma, \pi)$.

Huber was actually interested in groups with a compact fundamental domain, and used the lemma and the Weyl estimate (4.1) for such groups to get a result like Corollary 2 below. Since groups with compact fundamental domain have no continuous spectrum, he did not have to compute a scattering matrix, and so the Weyl estimate is simple. The proof of Huber's lemma is purely algebraic, depending only on the fact that the representation is irreducible; thus is carries over to this case as well.

Corollary 2. We have shown that

$$
\sum_{\substack{\lambda=(1 / 4)+r^{2} \\ 0<r<T}} m(\pi, \lambda) \sim \operatorname{dim} \pi \frac{\mu}{4 \pi} T^{2}, \quad \text { as } T \rightarrow \infty .
$$

For an example of one of these representation spaces, take the case of a prime level $q \equiv 1 \bmod 4$. Suppose $V$ is a space of wave forms in which the 
action of $\Gamma$ is equivalent to $\pi^{+}$in the notation of $\S 2$. Let $\chi_{1}$ be the quadratic character $\bmod q$. Then $\chi_{1}$ defines a representation of $\Gamma_{0}(q)$ in the usual way, by $\chi_{1}\left[\begin{array}{ll}a & b \\ c & d\end{array}\right]=\chi_{1}(d)$. The Frobenius reciprocity theorem says that the multiplicity of $\chi_{1}$ in the restriction of $\pi^{+}$to $\Gamma_{0}(q)$ is equal to the multiplicity of $\pi^{+}$in the induced representation of $\chi_{1}$ from $\Gamma_{0}(q)$. This multiplicity is equal to one by the lemmas in $\S 2$. Thus there is a unique one-dimensional space of wave forms $f$ in $V$ such that

$$
f\left(\frac{a z+b}{c z+d}\right)=\chi_{1}(d) f(z) \text {. }
$$

The same argument works in the case of a space of wave forms with the action equivalent to $\pi^{-}$. The wave forms constructed by Maass are of this type, with a Mellin transform as a Hecke $L$-function attached to a grossencharacter for $\mathbf{Q}(\sqrt{q})$. The eigenvalue depends on the grossencharacter. By the above argument, these wave forms must be contained in representation spaces equivalent to $\pi^{+}$or $\pi^{-}$. There are connections between the multiplicities $m\left(\pi^{ \pm}, 1 / 4\right)$ and class numbers for the real quadratic fields $\mathbf{Q}(\sqrt{q})$. A theorem analogous to Hecke's in the imaginary quadratic case (mentioned above) is proved in [12].

TABLE 1

\begin{tabular}{|c|c|c|c|c|}
\hline representation & dimension & $\begin{array}{l}\text { degree of } \\
\text { singularity }\end{array}$ & $\begin{array}{l}\text { case (i) } \\
g=\left[\begin{array}{ll}\alpha & \beta \\
y & \delta\end{array}\right]\end{array}$ & $\begin{array}{l}\text { case (ii) } \\
g=\left[\begin{array}{cc}\alpha & \beta \\
0 & \alpha^{-1}\end{array}\right]\end{array}$ \\
\hline$\hat{\chi}$ & $q+1$ & 2 & $\begin{array}{cc}0 & \frac{\chi(\gamma)}{\sqrt{q}} \\
\bar{\chi}(\gamma) & 0\end{array}$ & {$\left[\begin{array}{cc}\bar{\chi}(\alpha) & 0 \\
0 & \chi(\alpha)\end{array}\right.$} \\
\hline 1 & 1 & 1 & $\sqrt{q}$ & 1 \\
\hline$\theta$ & $q$ & 1 & $\frac{-1}{a}$ & 1 \\
\hline$\pi^{+}$ & $\frac{q+1}{2}$ & 1 & $\frac{1}{\sqrt{q}} \chi_{1}(\gamma)$ & $\chi_{1}(\alpha)$ \\
\hline$\pi^{-}$ & $\frac{q+1}{2}$ & 1 & $\frac{-1}{\sqrt{q}} \chi_{1}(\gamma)$ & $\chi_{1}(\alpha)$ \\
\hline
\end{tabular}

The first three columns list the singular representations of $S L(2, \mathbf{Z} / q)$ for prime $q$, their dimension, and degree of singularity. The last two columns show the relevant matrix coefficients $A_{g}^{\pi}$ for each of two cases.

\section{REFERENCES}

1. E. Hecke, Mathematische Werke, Vandenhoeck und Ruprecht, Göttingen, 1983.

2. D. Hejhal, The Selberg trace formula for $\operatorname{PSL}(2, \mathbf{R})$, Vol. II, Lecture Notes in Math., vol. 1001, Springer-Verlag, New York, 1983.

3. H. Huber, Über die Darstellungen der Automorphismengruppe einer Riemannschen fläche in den Eigenräumen des Laplace-operators. Comment. Math. Helv. 52 (1977), 177-184.

4. H. Kloosterman, The behavior of general theta functions under the modular group, and the characters of binary modular congruence groups. I, II, Ann. of Math. 47 (1946), 317-447. 
5. H. Maass, Über eine neue Art von nichtanalytischen automorphen Funktionen und die Bestimmung Dirichletscher Reihen durch Funktionalgleichungen, Math. Ann. 121 (1949), 141-183.

6. I. Piatetskii-Shapiro, Complex representations of $G L(2, K)$ for finite fields $K$, Contemp. Math., vol. 16, Amer. Math. Soc., Providence, R.I., 1980.

7. K. Prachar, Primzahlverteilung, Springer-Verlag, Berlin, 1957.

8. B. Randol, A remark on the multiplicity of the discrete spectrum of congruence subgroups, Proc. Amer. Math. Soc. 81 (1981), 339-340.

9. A. Selberg, Harmonic analysis and discontinuous groups in weakly symmetric Riemannian spaces with applications to Dirichlet series, J. Indian Math. Soc. 20 (1956), 47-87.

10. J.-P. Serre, Linear representations of finite groups, Graduate Texts in Math. 42, SpringerVerlag, New York, 1982.

11. J. Stopple, A functional equation for some Selberg zeta functions, Ph.D. dissertation, Univ. of California, San Diego, 1986.

12. __ Selberg zeta functions with virtual characters and the class number, Acta Arith. (to appear).

13. S. Tanaka, Construction and classification of irreducible representations of special linear group of the second order over a finite field, Osaka J. Math. 4 (1967), 65-84.

Department of Mathematics, University of California, Santa Barbara, California 93106 